\title{
Supporting Information: Stepwise solvation of an amino acid: The appearance of Zwitterionic
}

\section{structures}

Martine N. Blom, ${ }^{a}$ Isabelle Compagnon, ${ }^{a}$ Nick C. Polfer, ${ }^{a}$ Gert von Helden, ${ }^{b}$ Gerard Meijer,${ }^{b}$ Sándor Suhai, ${ }^{c}$ Béla Paizs ${ }^{\dagger c}$ and Jos Oomens $*^{a}$

${ }^{a}$ FOM Institute for Plasmaphysics “Rijnhuizen”, Edisonbaan 14, NL-3439 MN Nieuwegein, The Netherlands.

${ }^{b}$ Fritz-Haber-Institut der Max-Planck-Gesellschaft, Faradayweg 4-6, D-14195, Germany.

${ }^{\mathrm{c}}$ German Cancer Research Center, Im Neuenheimer Feld 580, D-69120 Heidelberg, Germany.

*Corresponding author: joso@ rijnhuizen.nl

${ }^{\dagger}$ Corresponding author for computations: $\underline{\text { b.paizs@ @ dkfz-heidelberg.de }}$

Figure S1. FT-IR spectra of $\mathrm{H}_{2} \mathrm{O}, \mathrm{D}_{2} \mathrm{O}, \mathrm{MeOH}$ and $\mathrm{MeOD}$ with (solid line) and without (dot line) tryptophan. The arrows indicate the position of the asymmetric $\mathrm{CO}_{2}^{-}$stretch for tryptophan in the different solvents. 


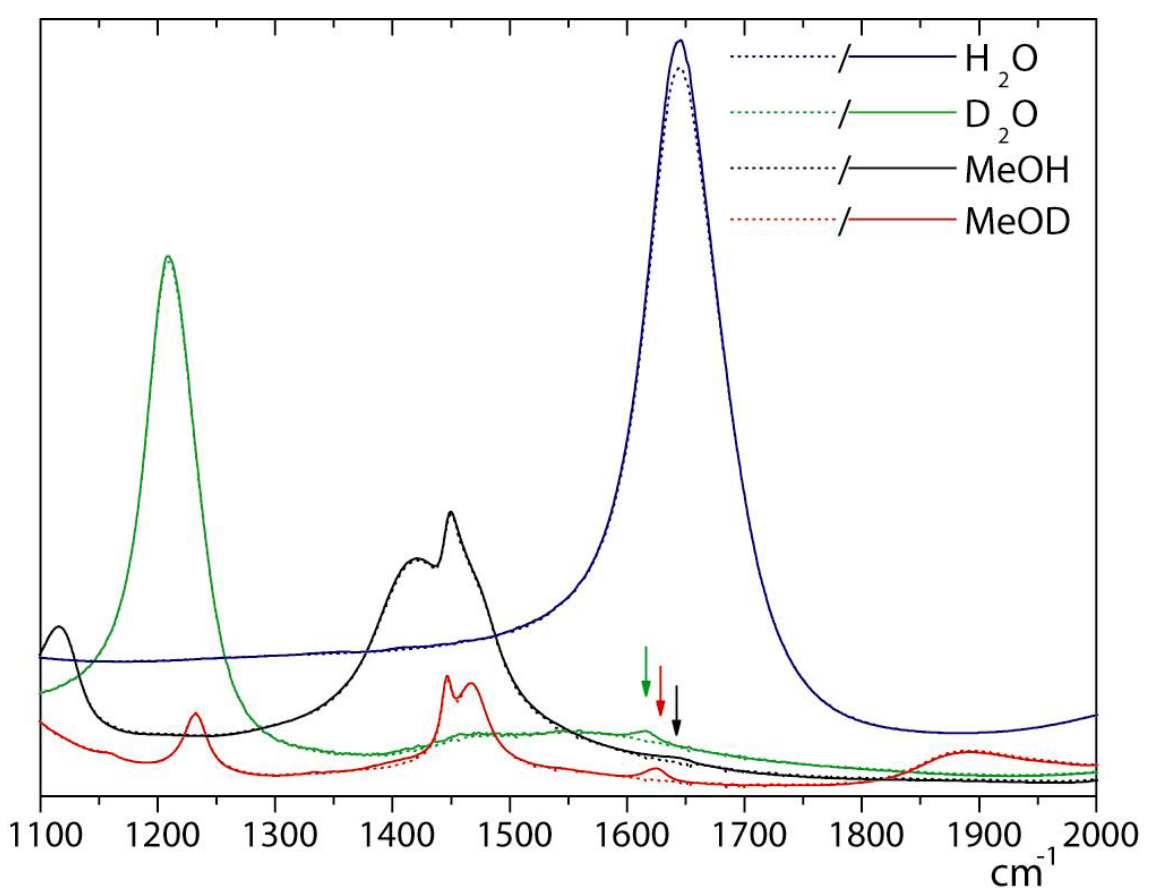


Table S1-S6. Summary of the performed $\operatorname{Trp}(\text { Wat })_{n}$ calculations. Geometry optimizations are carried out at the density functional theory level (B3LYP/6 $\left.-31+\mathrm{g}^{* *}\right)$ and the energies are refined using a single point calculation at the higher BSSE corrected MP2 level. The zero point corrected total energy $\mathrm{E}$ [Hartree] of each lowest energy structure (in brackets), as well as the relative energies $\Delta \mathrm{E}\left[\mathrm{kcal} \mathrm{mol}^{-1}\right]$ are given in the leftmost column. The geometry of each conformer is represented by its structure type $(\mathrm{N} 1, \mathrm{~N} 2, \mathrm{ZW})$ and the water binding sites on the tryptophan. The $\mathrm{CO}$ stretch frequency calculated at the density functional level (B3LYP/6-31+g**) are shown for $\operatorname{Trp}\left(\mathrm{D}_{2} \mathrm{O}\right)_{n}$ in the rightmost column (scaled by a factor 0.987).

Table S1. Trp(Wat $)_{1}$

\begin{tabular}{cccc}
\hline$\Delta \mathrm{E} / \mathrm{kcal} / \mathrm{mol}(\mathrm{E} /$ Hartree $)$ & structure type & Water binding sites & $v(\mathrm{CO}) \operatorname{Trp}\left(\mathrm{D}_{2} \mathrm{O}\right)_{1}$ \\
\hline$+0.0(-760,41384)$ & $\mathrm{N} 1$ & $\mathrm{CO}, \mathrm{COH}$ & 1748 \\
+0.27 & $\mathrm{~N} 1$ & $\mathrm{CO}, \mathrm{COH}$ & 1748 \\
+0.58 & $\mathrm{~N} 1$ & $\mathrm{CO}, \mathrm{COH}$ & 1748 \\
+0.61 & $\mathrm{~N} 1$ & $\mathrm{CO}, \mathrm{COH}$ & 1749 \\
+0.66 & $\mathrm{~N} 1$ & $\mathrm{CO}, \mathrm{COH}$ & 1741 \\
+1.01 & $\mathrm{~N} 1$ & $\mathrm{CO}, \mathrm{COH}$ & 1734 \\
+1.12 & $\mathrm{~N} 2$ & $\mathrm{CO}$ & 1803 \\
+1.25 & $\mathrm{~N} 1$ & $\mathrm{CO}, \mathrm{COH}$ & 1731 \\
+1.34 & $\mathrm{~N} 1$ & $\mathrm{CO}, \mathrm{COH}$ & 1758 \\
+1.42 & $\mathrm{~N} 1$ & $\mathrm{CO}, \mathrm{COH}$ & 1752 \\
+1.53 & $\mathrm{~N} 1$ & $\mathrm{CO}, \mathrm{COH}$ & 1733 \\
+1.58 & $\mathrm{~N} 1$ & $\mathrm{CO}, \mathrm{COH}$ & 1733 \\
+1.68 & $\mathrm{~N} 2$ & $\mathrm{CO}$ & 1799 \\
+1.74 & $\mathrm{~N} 1$ & $\mathrm{CO}, \mathrm{COH}$ & 1747 \\
+1.78 & $\mathrm{~N} 2$ & indole NH & 1823
\end{tabular}




$\begin{array}{cccc}+1.89 & \mathrm{~N} 1 & \mathrm{CO}, \mathrm{COH} & 1748 \\ +1.89 & \mathrm{~N} 1 & \mathrm{CO}, \mathrm{COH} & 1736 \\ +1.98 & \mathrm{~N} 2 & \mathrm{CO} & 1798 \\ +11.86 & \mathrm{ZW} & \mathrm{CO}, \mathrm{NH}_{3} & 1756 \\ +12.04 & \mathrm{ZW} & \mathrm{CO}, \mathrm{NH}_{3} & 1758 \\ +12.49 & \mathrm{ZW} & \mathrm{CO}, \mathrm{NH}_{3} & 1756 \\ +12.76 & \mathrm{ZW} & \mathrm{CO}, \mathrm{NH}_{3} & 1753\end{array}$


Table S2. $\operatorname{Trp}(\text { Wat })_{2}$

\begin{tabular}{cccc}
\hline$\Delta \mathrm{E} / \mathrm{kcal} / \mathrm{mol}(\mathrm{E} /$ Hartree $)$ & structure type & Water binding sites & $v(\mathrm{CO}) \operatorname{Trp}\left(\mathrm{D}_{2} \mathrm{O}\right)_{2}$ \\
\hline$+0.0(-836.63892)$ & $\mathrm{N} 1$ & $\mathrm{CO}, \mathrm{COH}$ & 1737 \\
+0.05 & $\mathrm{~N} 2$ & $\mathrm{CO}$, indole $\mathrm{NH}$ & 1792 \\
+0.59 & $\mathrm{~N} 2$ & $\mathrm{CO}$, indole $\mathrm{NH}$ & 1790 \\
+1.50 & $\mathrm{~N} 1$ & $\mathrm{CO}, \mathrm{COH}$ & 1734 \\
+1.70 & $\mathrm{~N} 1$ & $\mathrm{CO}, \mathrm{COH}$ & 1744 \\
+1.73 & $\mathrm{~N} 1$ & $\mathrm{CO}, \mathrm{COH}$ & 1736 \\
+1.79 & $\mathrm{~N} 1$ & $\mathrm{CO}, \mathrm{COH}$ & 1735 \\
+1.84 & $\mathrm{~N} 1$ & $\mathrm{CO}, \mathrm{COH}$ & 1718 \\
+1.89 & $\mathrm{~N} 1$ & $\mathrm{CO}, \mathrm{COH}$ & 1732 \\
+1.94 & $\mathrm{~N} 1$ & $\mathrm{CO}, \mathrm{COH}$ & 1738 \\
+1.97 & $\mathrm{~N} 1$ & $\mathrm{CO}, \mathrm{COH}$ & 1716 \\
+9.00 & $\mathrm{ZW}$ & $\mathrm{CO}, \mathrm{NH}$ & 1751 \\
+10.40 & $\mathrm{ZW}$ & $\mathrm{CO}, \mathrm{NH}$ & 1745 \\
\hline
\end{tabular}


Table S3. Trp(Wat $)_{3}$

\begin{tabular}{cccc}
\hline$\Delta \mathrm{E} / \mathrm{kcal} / \mathrm{mol}(\mathrm{E} /$ Hartree $)$ & structure type & Water binding sites & $v(\mathrm{CO}) \operatorname{Trp}\left(\mathrm{D}_{2} \mathrm{O}\right)_{3}$ \\
\hline$+0.0(-912.86080)$ & $\mathrm{N} 2$ & $\mathrm{CO}$, indole NH & 1790 \\
+0.02 & $\mathrm{~N} 1$ & $\mathrm{CO}, \mathrm{COH}$ & 1733 \\
+0.33 & $\mathrm{~N} 1$ & $\mathrm{CO}, \mathrm{COH}, \mathrm{NH}_{2}$ & 1737 \\
+0.57 & $\mathrm{~N} 1$ & $\mathrm{CO}, \mathrm{COH}$ & 1739 \\
+0.58 & $\mathrm{~N} 1$ & $\mathrm{CO}, \mathrm{COH}$ & 1728 \\
+0.90 & $\mathrm{~N} 1$ & $\mathrm{CO}, \mathrm{COH}$ & 1735 \\
+1.14 & $\mathrm{~N} 1$ & $\mathrm{CO}, \mathrm{COH}$ & 1733 \\
+1.14 & $\mathrm{~N} 1$ & $\mathrm{CO}, \mathrm{COH}$ & 1741 \\
+1.54 & $\mathrm{~N} 1$ & $\mathrm{CO}, \mathrm{COH}$, indole NH & 1745 \\
+5.79 & $\mathrm{ZW}$ & $\mathrm{CO}, \mathrm{CO}$, indole $\mathrm{NH}$ & 1721 \\
+6.79 & $\mathrm{ZW}$ & $\mathrm{CO}, \mathrm{CO}, \mathrm{NH}{ }_{3}$ & 1708 \\
\hline
\end{tabular}


Table S4. $\operatorname{Trp}(\text { Wat })_{4}$

\begin{tabular}{|c|c|c|c|}
\hline$\Delta \mathrm{E} / \mathrm{kcal} / \mathrm{mol}$ (E / Hartree) & structure type & Water binding sites & $v(\mathrm{CO}) \operatorname{Trp}\left(\mathrm{D}_{2} \mathrm{O}\right)_{4}$ \\
\hline$+0.00(-989.084392)$ & N1 & $\mathrm{CO}, \mathrm{COH}$, indole $\mathrm{NH}$ & 1742 \\
\hline+0.68 & N1 & $\mathrm{CO}, \mathrm{COH}$, indole $\mathrm{NH}$ & 1714 \\
\hline+0.93 & N1 & $\mathrm{CO}, \mathrm{COH}$, indole $\mathrm{NH}$ & 1724 \\
\hline+0.93 & N1 & $\mathrm{CO}, \mathrm{COH}$, indole $\mathrm{NH}$ & 1743 \\
\hline+1.23 & $\mathrm{~N} 1$ & $2 * \mathrm{CO}, \mathrm{COH}$ & 1707 \\
\hline+1.29 & N1 & $\mathrm{CO}, \mathrm{COH}$ & 1759 \\
\hline+1.30 & N1 & $\mathrm{CO}, \mathrm{COH}$ & 1711 \\
\hline+1.31 & N1 & $\mathrm{CO}, \mathrm{COH}$ & 1670 \\
\hline+1.45 & N1 & $\mathrm{COH}$, indole $\mathrm{NH}, \mathrm{NH}_{2}$ & 1773 \\
\hline+3.24 & $\mathrm{~N} 2$ & $\mathrm{CO}$, indole $\mathrm{NH}$ & 1791 \\
\hline+3.22 & $\mathrm{~N} 2$ & $\mathrm{CO}$, indole $\mathrm{NH}$ & 1797 \\
\hline+5.41 & $\mathrm{ZW}$ & $\mathrm{CO}, 2 * \mathrm{CO}, 2 * \mathrm{NH}_{3}$ & 1686 \\
\hline+5.43 & $\mathrm{ZW}$ & $\mathrm{CO}, \mathrm{CO}, \mathrm{NH}_{3}$ & 1700 \\
\hline+5.56 & ZW & $1 * \mathrm{CO}, 2 * \mathrm{CO}, 2 * \mathrm{NH}_{3}$ & 1687 \\
\hline+5.58 & ZW & $1 * \mathrm{CO}, 2 * \mathrm{CO}, 2 * \mathrm{NH}_{3}$ & 1685 \\
\hline+6.21 & ZW & $1 * \mathrm{CO}, 2 * \mathrm{CO}, 2 * \mathrm{NH}_{3}$ & 1688 \\
\hline+6.28 & ZW & $1 * \mathrm{CO}, 2 * \mathrm{CO}, 2 * \mathrm{NH}_{3}$ & 1687 \\
\hline
\end{tabular}


Table S5. Trp(Wat $)_{5}$

\begin{tabular}{|c|c|c|c|}
\hline$\Delta \mathrm{E} / \mathrm{kcal} / \mathrm{mol}$ (E / Hartree) & structure type & Water binding sites & $\mathrm{v}(\mathrm{CO}) \operatorname{Trp}\left(\mathrm{D}_{2} \mathrm{O}\right)_{5}$ \\
\hline$+0.0(-1065.30690)$ & N1 & $\mathrm{CO}, \mathrm{COH}$, indole $\mathrm{NH}$ & 1726 \\
\hline+0.13 & N1 & $\mathrm{CO}, \mathrm{COH}$, indole $\mathrm{NH}$ & 1703 \\
\hline+0.24 & N1 & $\mathrm{CO}, \mathrm{COH}$, indole $\mathrm{NH}$ & 1752 \\
\hline+0.34 & N1 & $\mathrm{CO}, \mathrm{COH}$, indole $\mathrm{NH}, \mathrm{NH}_{2}$ & 1737 \\
\hline+0.36 & N1 & $\mathrm{CO}, \mathrm{COH}$, indole $\mathrm{NH}$ & 1726 \\
\hline+0.55 & N1 & $\mathrm{CO}, \mathrm{COH}$, indole $\mathrm{NH}$ & 1718 \\
\hline+0.65 & N1 & $\mathrm{CO}, \mathrm{COH}, \mathrm{COH}$, indole $\mathrm{NH}$ & 1709 \\
\hline+0.69 & N1 & $\mathrm{CO}, \mathrm{COH}$, indole $\mathrm{NH}$ & 1725 \\
\hline+0.72 & N1 & $\mathrm{CO}, \mathrm{COH}$, indole $\mathrm{NH}$ & 1755 \\
\hline+0.92 & N1 & $\mathrm{CO}, \mathrm{COH}, \mathrm{NH}_{2}$ & 1743 \\
\hline+0.94 & N1 & $2 * \mathrm{CO}, \mathrm{COH}$ & 1699 \\
\hline+0.95 & N1 & $\mathrm{CO}, \mathrm{COH}, \mathrm{NH}_{2}$ & 1760 \\
\hline+1.14 & N1 & $\mathrm{CO}, \mathrm{COH}$ & 1751 \\
\hline+1.42 & N1 & $\mathrm{CO}, \mathrm{COH}$, indole $\mathrm{NH}$ & 1746 \\
\hline+1.5 & N1 & $\mathrm{CO}, \mathrm{COH}$, indole $\mathrm{NH}$ & 1739 \\
\hline+3.51 & $\mathrm{~N} 2$ & $\mathrm{CO}, \mathrm{COH}$, indole $\mathrm{NH}$ & 1807 \\
\hline+3.99 & $\mathrm{~N} 2$ & $\mathrm{CO}, \mathrm{NH}_{2}$, indole $\mathrm{NH}$ & 1790 \\
\hline+2.91 & $\mathrm{ZW}$ & $\mathrm{NH}_{3}, \mathrm{NH}_{3}, 2 * \mathrm{CO}, \mathrm{CO}$ & 1684 \\
\hline+3.62 & $\mathrm{ZW}$ & $\mathrm{NH}_{3}, \mathrm{NH}_{3}, 2 * \mathrm{CO}, \mathrm{CO}$, indole $\mathrm{NH}$ & 1682 \\
\hline+3.99 & $\mathrm{ZW}$ & $\mathrm{NH}_{3}, \mathrm{NH}_{3}, 2 * \mathrm{CO}, \mathrm{CO}$ & 1697 \\
\hline+4.29 & $\mathrm{ZW}$ & $\mathrm{NH}_{3}, \mathrm{NH}_{3}, 2 * \mathrm{CO}, \mathrm{CO}$ & 1672 \\
\hline+4.32 & $\mathrm{ZW}$ & $\mathrm{NH}_{3}, \mathrm{NH}_{3}, 2 * \mathrm{CO}, \mathrm{CO}$ & 1691 \\
\hline+4.37 & $\mathrm{ZW}$ & $\mathrm{NH}_{3}, \mathrm{NH}_{3}, 2 * \mathrm{CO}, \mathrm{CO}$ & 1676 \\
\hline+4.54 & $\mathrm{ZW}$ & $\mathrm{NH}_{3}, \mathrm{NH}_{3}, 2 * \mathrm{CO}, \mathrm{CO}$ & 1672 \\
\hline+4.54 & $\mathrm{ZW}$ & $\mathrm{NH}_{3}, \mathrm{NH}_{3}, 2 * \mathrm{CO}, \mathrm{CO}$ & 1672 \\
\hline
\end{tabular}




$\begin{array}{llcc}+4.58 & \mathrm{ZW} & \mathrm{NH}_{3}, \mathrm{NH}_{3}, 2 * \mathrm{CO}, \mathrm{CO} & 1675 \\ +4.62 & \mathrm{ZW} & \mathrm{NH}_{3}, \mathrm{NH}_{3}, 2 * \mathrm{CO}, \mathrm{CO} & 1684 \\ +4.65 & \mathrm{ZW} & \mathrm{NH}_{3}, \mathrm{NH}_{3}, 2 * \mathrm{CO}, 2 * \mathrm{CO} & 1670 \\ +4.72 & \mathrm{ZW} & \mathrm{NH}_{3}, \mathrm{NH}_{3}, 2 * \mathrm{CO}, \mathrm{CO} & 1687 \\ +4.74 & \mathrm{ZW} & \mathrm{NH}_{3}, \mathrm{NH}_{3}, 2 * \mathrm{CO}, \mathrm{CO} & 1674 \\ +4.75 & \mathrm{ZW} & \mathrm{NH}_{3}, \mathrm{NH}_{3}, 2 * \mathrm{CO}, \mathrm{CO} & 1691 \\ +4.79 & \mathrm{ZW} & \mathrm{NH}_{3}, \mathrm{CO}, \mathrm{CO}, \text { indole } \mathrm{NH} & 1700 \\ +4.81 & \mathrm{ZW} & \mathrm{NH}_{3}, \mathrm{NH}_{3}, 2 * \mathrm{CO}, \mathrm{CO} & 1675 \\ +4.86 & \mathrm{ZW} & \mathrm{NH}_{3}, \mathrm{NH}_{3}, 2 * \mathrm{CO}, \mathrm{CO} & 1691\end{array}$


Table S6. Trp $(\text { Wat })_{6}$

\begin{tabular}{|c|c|c|c|}
\hline$\Delta \mathrm{E} / \mathrm{kcal} / \mathrm{mol}(\mathrm{E} / \mathrm{Hartree})$ & structure type & Water binding sites & $v(\mathrm{CO}) \operatorname{Trp}\left(\mathrm{D}_{2} \mathrm{O}\right)_{5}$ \\
\hline$+0.0(-1141.52958)$ & N1 & $2 * \mathrm{CO}, \mathrm{COH}$ & 1696 \\
\hline+0.42 & N1 & $2 * \mathrm{CO}, \mathrm{COH}$, indole $\mathrm{NH}$ & 1716 \\
\hline+0.63 & N1 & $\mathrm{CO}, \mathrm{COH}, \mathrm{COH}$, indole $\mathrm{NH}$ & 1753 \\
\hline+0.67 & N1 & $\mathrm{CO}, \mathrm{COH}$, indole $\mathrm{NH}$ & 1730 \\
\hline+0.85 & N1 & $\mathrm{CO}, \mathrm{COH}, \mathrm{NH} 2$, indole $\mathrm{NH}$ & 1753 \\
\hline+0.89 & N1 & $\mathrm{CO}, \mathrm{COH}, \mathrm{COH}$, indole $\mathrm{NH}$ & 1721 \\
\hline+1.10 & N1 & $\mathrm{CO}, \mathrm{COH}, \mathrm{COH}$, indole $\mathrm{NH}$ & 1736 \\
\hline+1.11 & N1 & $\mathrm{CO}, \mathrm{COH}$, indole $\mathrm{NH}$ & 1718 \\
\hline+1.15 & N1 & $\mathrm{CO}, \mathrm{COH}$, indole $\mathrm{NH}$ & 1699 \\
\hline+1.24 & N1 & $\mathrm{CO}, \mathrm{COH}$, indole $\mathrm{NH}$ & 1734 \\
\hline+1.26 & N1 & $2 * \mathrm{CO}, \mathrm{COH}$, indole $\mathrm{NH}$ & 1699 \\
\hline+1.40 & N1 & $\mathrm{CO}, \mathrm{COH}, \mathrm{COH}$, indole $\mathrm{NH}$ & 1722 \\
\hline+1.40 & N1 & $\mathrm{CO}, \mathrm{COH}$, indole $\mathrm{NH}$ & 1754 \\
\hline+1.42 & N1 & $2 * \mathrm{CO}, \mathrm{COH}$ & 1706 \\
\hline+1.42 & N1 & $\mathrm{CO}, \mathrm{COH}, \mathrm{COH}$ & 1749 \\
\hline+1.44 & N1 & $\mathrm{CO}, \mathrm{OH}$, indole $\mathrm{NH}$ & 1703 \\
\hline+1.45 & N1 & $2 * \mathrm{CO}, \mathrm{COH}$, indole $\mathrm{NH}$ & 1713 \\
\hline+1.16 & ZW & $2 * \mathrm{CO}, \mathrm{CO}, \mathrm{NH}_{3}$, indole $\mathrm{NH}$ & 1694 \\
\hline+1.26 & ZW & $2 * \mathrm{CO}, \mathrm{CO}, \mathrm{NH}_{3}, \mathrm{NH}_{3}$ & 1690 \\
\hline+1.67 & ZW & $\mathrm{CO}, \mathrm{CO}, \mathrm{NH}_{3}, \mathrm{NH}_{3}$ & 1691 \\
\hline+2.04 & $\mathrm{ZW}$ & $2 * \mathrm{CO}, \mathrm{CO}, \mathrm{NH}_{3}, \mathrm{NH}_{3}$ & 1676 \\
\hline+2.15 & $\mathrm{ZW}$ & $\underset{\mathrm{NH}}{\mathrm{CO}, 2 * \mathrm{CO}, \mathrm{NH}_{3}, \mathrm{NH}_{3} \text {, indole }}$ & 1677 \\
\hline+2.38 & ZW & $\mathrm{CO}, \mathrm{CO}, \mathrm{NH}_{3}$ & 1696 \\
\hline+2.79 & $\mathrm{ZW}$ & $\mathrm{CO}, 2 * \mathrm{CO}, \mathrm{NH}_{3}, \mathrm{NH}_{3}$ & 1664 \\
\hline+2.82 & ZW & $\mathrm{CO}, 2 * \mathrm{CO}, \mathrm{NH}_{3}, \mathrm{NH}_{3}, \mathrm{NH}_{3}$ & 1685 \\
\hline
\end{tabular}


